\title{
La Gestión del Conocimiento y la Innovación Hacia el Desarrollo Local Sostenible: Experiencia del Proyecto Piloto del Municipio la Palma con la Universidad de Pinar del Río, República de Cuba
}

Knowledge Management and Innovation Towards Sustainable Local Development: Experience of the Pilot municipality of La Palma with the University of Pinar del Rio, Republic of Cuba

Mayra Casas Vilardell

\section{Resumen}

Se presenta la experiencia positiva de un proyecto piloto universitario, de la Universidad de Pinar del Río "Hermanos Saíz Monte de Oca", de la República de Cuba, en el municipio La Palma, a partir de la concepción de un proyecto universitario, que integra con la actuación colectiva de todo el equipo que lo conforma, la gestión del conocimiento y la innovación, en función del desarrollo local sostenible en el territorio, coordinado a través de un Comité gestor Gobierno local-Universidad. Se destaca una actuación universitaria que reconoce desde la base inicial de la propuesta, su rol como facilitador, y que incluye acciones de capacitación, colaboración, y desarrollo de investigaciones, a la vez que subraya la importancia que en todo este proceso tiene significar el rol protagónico del gobierno local del territorio, su liderazgo, autoridad, responsabilidad y atención permanente a la toma de decisiones. Una importante fortaleza del proyecto, la constituye desarrollar con carácter interdisciplinar las acciones de corte social, económico y ecológico que se abordan con un enfoque integral.

Palabras-clave: Gestión del Conocimiento. Innovación. Desarrollo Local.

\begin{abstract}
This presents the positive experience of a university pilot project of the University of Pinar del Rio "Hermanos Saíz Montes de Oca", the Republic of Cuba, in the municipality of La Palma, from the conception of auniversity project, which integrates the collective action of all the equipment that forms, knowledge management and innovation, according to local sustainable development in the area, coordinated through a steering committee of local GovernmentUniversity. Performance highlights university recognized from the initial basis of the proposal, its role as facilitator and includes training activities, collaborative research and development, while stressing the importance that this process has meantthe role leading local government planning, leadership, authority, accountability and continued focus on decision making. A major strength of the project, is todevelop interdisciplinary activities of a social, economic and ecological that are addressed with a holistic approach.
\end{abstract}

Keywords: Knowledge Management. Innovation. LocalDevelopment.

\section{Introducion}

El Ministerio de Educación Superior cubano ha llamado a su claustro a desarrollar una actuación más dinámica en su encargo universitario y en esa dirección, fundamentar y reorientar un camino más proactivo, que fundamente no solo el marco teórico de las propuestas, sino que acompañe sus procesos de aplicación en la práctica, dando prioridad a aquellas líneas en que el territorio y el país tienen las mayores necesidades.

La "Nueva Universidad" del siglo XXI es diferente a la del siglo pasado, en ello inciden múltiples factores y sin dudas con el proceso de universalización de la educación superior, los actores locales cobran un realce muy especial al permitir mayor dinamismo en la vinculación teoría-práctica, transitar del conocimiento empírico al conocimiento construido, a una práctica más aplicada a la teoría, donde se propicia el diálogo de saberes entre la academia y el conocimiento popular y resalta la máxima de la sostenibilidad que llama al pensamiento global y a la actuación local. 
La propuesta de la universidad contemporánea llama a la fusión de la ciencia y la experiencia, transitar del pensamiento a la realidad, de la hipótesis a la certeza, lo cual no significa deslinde académico, sino una apropiada articulación de saberes: científicos y populares, y el conocimiento y su aplicabilidad se vinculan estrechamente a modelos de gestión adecuados al contexto local en una lógica sistémica que potencie la participación de sus actores, y la articulación en redes locales, nacionales e internacionales que favorezcan de forma constante y permanente el proceso de innovación.

La universidad es un espacio de producción y difusión del conocimiento y la cultura, en un siglo donde el debate sobre el desarrollo, se centra en el conocimiento como el factor más dinámico del mismo. La sociedad actual, desde su evidente complejidad, precisa de un alto grado de conocimiento y de mayor capacidad de los individuos involucrados en los procesos, y ello la convierte, en una sociedad cada vez más crítica y exigente con la institución que tiene ese importante encargo.

<<La "Nueva Universidad", ofrece oportunidades inéditas de poner los conocimientos al servicio de la solución de los problemas territoriales, creando un nuevo actor colectivo del conocimiento, promotor del aprendizaje, potencialmente volcado a la innovación, capaz de favorecer la creación de competencias para la asimilación/creación de tecnologías y saberes de significación social, siempre en vínculo con los restantes actores del territorio: políticos, administrativos, educativos, etc. > > (Núñez, 2002) ${ }^{1}$.

El desarrollo local requiere del desarrollo de la universidad y viceversa. Esto implica una complejización de la misión de las universidades y su articulación, como factor de la constitución e integración de actores locales para el desarrollo y como mediadora del conocimiento científico que requiere el desarrollo local participativo y sostenible. (Coraggio, 2002) ${ }^{2}$

Si el desarrollo está centrado en el conocimiento, la sociedad local en desarrollo se convierte en una red de comunidades de aprendizaje, donde se aprende no sólo estudiando, sino mediante prácticas colectivas reflexivas; así éstas comunidades se piensan a sí mismas y se proyectan hacia el futuro posicionándose en el contexto del municipio, la provincia, el país, y el mundo que las rodea. Las universidades e instituciones científicas deben privilegiar estas prácticas que implican el desarrollo de capacidades locales, facilitando los procesos y adaptándose a sus peculiaridades.

$<<$ Un elemento distintivo del desarrollo local para Cuba es que constituye un complemento necesario a las políticas y objetivos nacionales. Las iniciativas de desarrollo local deben revitalizar el vínculo entre las autoridades centrales y la administración provincial y municipal, brindando mayor protagonismo a los actores locales en la búsqueda de soluciones a sus propios problemas, teniendo siempre en cuenta la correspondencia con dichos lineamientos nacionales. En este sentido, existen importantes oportunidades para el avance de procesos endógenos de desarrollo local, que lejos de contraponerse al nivel central, se complementen con la planificación nacional. >> (González, A. y Samper, Y., 2006) ${ }^{3}$

Desde septiembre del 2009 se inicia por indicación del Ministerio de Educación Superior (MES), la experiencia de un proyecto piloto universitario, concibiéndose el de la Universidad de Pinar del Río "Hermanos Saíz Monte de Oca", en el municipio La Palma, a partir de la concepción del mismo como un proyecto universitario, que integrara a todas las facultades, centros de estudios y Sedes universitarias municipales, a partir de la actuación colectiva integrada de todo el equipo que lo conforma y que se organizó a partir de un Comité gestor Gobierno-Universidad, con el objetivo de articular la gestión del conocimiento y la innovación en función del desarrollo local sostenible en ese municipio.

Resulta necesario destacar el rol protagónico del Gobierno del territorio, su liderazgo, autoridad, responsabilidad y actuación permanente en la organización y la toma de decisiones, y como en el caso de esta experiencia, el acompañamiento al trabajo de la Universidad y el Gobierno, contó con el respaldo permanente del Partido del municipio, que favoreció de manera integral la prioridad a la atención de la experiencia y sin dudas garantizó del éxito obtenido en esta importante etapa inicial del trabajo desarrollado durante el año 2010.

\section{¿Por qué el municipio La Palma?}

La elección del municipio piloto, respondió en primer lugar a una decisión del Gobierno Provincial en Pinar del Río, de desarrollar la experiencia en un municipio con características singulares en el territorio, por constituir el mismo un ecosistema frágil de montaña y ser además uno de los cinco municipios del país elegido para llevar adelante una experiencia sobre la Iniciativa Municipal de Desarrollo Local conducida por el Ministerio de Economía y Planificación desde el 2004, con vistas a la identificación de potencialidades de desarrollo endógeno en el ámbito municipal y las posibles soluciones a los principales problemas que afectan al territorio, incluyendo al sector no estatal como agente dinamizador del desarrollo agropecuario. 
La Universidad de Pinar del Río ratificó la decisión, unida a una serie de atributos del municipio que confirmaban la selección del territorio para la experiencia. En primer lugar, en este municipio se gestó el primer embrión universitario en la provincia con la Sede de Cajálbana, en este territorio está enclavada la Facultad de Montaña (una de las cinco del país), existe en el mismo una Unidad Docente y su Sede Universitaria Municipal, hoy devenida en Centro Universitario Municipal ostenta una destacada y reconocida actividad universitaria.

El municipio La Palma cuenta con fortalezas indiscutibles para convertirse en un polígono experimental, dirigido al acercamiento paulatino hacia el desarrollo sostenible de la montaña cubana, en el que confluyen satisfactoriamente, unidos a lo anterior, muchos factores con una visión de colaboración responsable, encabezados por el PCC y el Gobierno del municipio y donde una serie de instituciones como un Politécnico Forestal, la Junta Coordinadora del Plan Turquino, el Órgano del CITMA de Montaña, el Cuerpo de Guardabosques, la Empresa Forestal Integral, el Servicio Estatal Forestal, entre otras, unido a resultar durante mas de tres décadas objeto del estudio de investigaciones universitarias desarrolladas por diferentes Centros de Estudio e investigación de la universidad (Centro de Estudios Forestales, Centro de Estudios de Medio ambiente y Recursos Naturales, Centro de Estudios de Gerencia, Desarrollo Local y Turismo, Grupo de Agroecología, Centro de Estudios de Desarrollo Cooperativo y Comunitario, entre otros) brindan un marco que potencia y favorece la puesta en práctica de las soluciones obtenidas y las propuestas derivadas de numerosos estudios desarrollados en tesis doctorales, maestrías, diplomados, tesis de grado, jornadas científicas, forum de ciencia y técnica y encuentros y talleres de experiencias populares campesinas, validadas satisfactoriamente en el entorno palmero.

Este municipio ha sido objeto de estudio de cientos de investigaciones en el ámbito natural, social y económico y varios proyectos ramales y territoriales abordan su problemática desde diferentes dimensiones, por otra parte las experiencias dirigidas por varios centros de estudios de la universidad abordan los temas objeto de interés y uno de los proyectos territoriales coordinados por el Centro de Estudios de Medio Ambiente y Recursos naturales de la universidad, se orienta a desarrollar un Sistema de Gestión pertinente del conocimiento y la innovación en sus dimensiones: natural, social y económica para el manejo de los recursos ambientales en el macizo montañoso del municipio La Palma, todo lo cual fue considerado como apropiado punto de partida para la coordinación del trabajo inicial del proyecto, reconociendo que la conducción de la experiencia piloto sería rotatoria entre las áreas de investigación, en conformidad a las diferentes fases de tránsito del proyecto piloto.

La necesidad de activar el proceso de introducción y generalización de resultados y el enmarcarse los mismos dentro de las prioridades de la investigación científica y la innovación en Pinar del Río hasta el 2010, representadas en las áreas de: Desarrollo sostenible de la montaña, aprovechamiento de recursos energéticos, producción de alimentos, medio ambiente y desarrollo sostenible, defensa, recuperación ganadera, producción tabacalera, aprovechamiento de recursos hídricos, salud y sociedad pinareña, ratifican la indiscutible prioridad que asume la investigación integrada que se propone y que suma también otra importante fortaleza, la de desarrollar transdisciplinarmente las acciones del proyecto con implicaciones de corte social, económico y ecológico que reclaman la visión de los problemas con un enfoque integral.

Se destaca una actuación universitaria que reconoce desde la base inicial de la concepción de la propuesta de proyecto, su rol como facilitador, y que incluye acciones de capacitación, acompañamiento, respaldo, colaboración, desarrollo de investigaciones y de innovaciones, a la vez que subraya la importancia que en todo este proceso tiene significar el rol protagónico del gobierno del territorio, su liderazgo, autoridad, responsabilidad y atención permanente a la organización y la toma de decisiones.

Destaca en el período 2009-2010 la sistematicidad del trabajo, la estabilidad del colectivo como grupo gestor de la UPR, en el caso del municipio se trabajó con respaldo del PCC, y el liderazgo del gobierno municipal, un excelente trabajo coordinador de la Sede Universitaria Municipal de La Palma y la implicación plena del grupo de actores del territorio, donde se ratificó desde su inicio el protagonismo que le correspondería asumir al Consejo de Administración Municipal en su desarrollo.

\section{Conclusiones}

Sin lugar a dudas, el desarrollo local requiere activismo universitario y ello implica una complejización de su misión y la apropiada articulación como factor de la constitución e integración de actores locales, como mediadora del conocimiento científico que se requiere.

Desarrollar una sociedad requiere aprender a aprender de su propia práctica colectiva, una sociedad con instituciones y personas capaces de seguir aprendiendo y aplicando ese conocimiento con sabiduría, de acuerdo a una racionalidad real y objetiva que priorice la calidad de vida 
intergeneracional y proteja los recursos ambientales que se lo permiten, subordinando el crecimiento y la acumulación a la necesidad de lograr ese objetivo estratégico.

La "nueva universidad" tiene que potenciar su rol como productora de conocimientos, reglas y valores, a partir de sus propias experiencias, reflexiones e investigaciones y convertir el contexto local en su campo de prácticas fundamental, en su espacio de generalización de experiencias, en su campo de innovación. No se trata solamente de la vinculación interpersonal cotidiana con otros miembros de la sociedad, sino la rearticulación del conocimiento científico, donde la ciencia y la investigación desarrollarán un papel fundamental, si contribuyen a predecir que la realidad local puede transformarse en la aspiración deseada por la sociedad, y participar en el proceso de determinación de objetivos y procedimientos para lograrlo. En esa dirección, encierra la universidad cubana su contribución directa como facilitadora de un auténtico proceso de aproximación al desarrollo local sostenible.

\section{Notas}

\footnotetext{
${ }^{1}$ Núñez. J. (2006) "La gestión del conocimiento, la Ciencia y la Tecnología en La Nueva Universidad: una aproximación conceptual" con L. F. Montalvo e I. Pérez Ones, en La Nueva Universidad Cubana y su contribución a la Universalización del conocimiento; Hernández, D y Benítez, F (Comps.), Editorial Félix Varela, La Habana.

2 Coraggio, J.L. 2002. Ponencia presentada en el Seminario Internacional .La educación superior y las nuevas tendencias.,organizado por el Consejo Nacional de Educación Superior (CONESUP), UNESCO y el CIESPAL.

${ }^{3}$ González, A. y Samper, Y. (2006). Iniciativa municipal para el desarrollo local. Tesis en opción a la Licenciatura en Economía, p.3, Facultad de Economía, Universidad de la Habana, Ciudad de la Habana
}

\section{Referências}

CAÑO, M. "Cuba, Desarrollo Local en los 90 ", del libro "Desarrollo local y descentralización en el contexto urbano", en: Tercer Taller de Desarrollo Urbano y Participación celebrado en diciembre de 1998, Universidad de la Habana, Facultad de Filosofía e Historia. Editorial Taller de Impresión del Movimiento Cubano por la Paz. 2000.

CASTILLO, L. y Pozo, J. M. “Desarrollo Local y Microcrédito.” en: Revista Economía y Desarrollo. Edición Especial. Vol. 136. La Habana, 2004.

CORAGGIO, J. L. Ponencia presentada en el Seminario Internacional. La educación superior y las nuevas tendencias, organizado por el Consejo Nacional de Educación Superior (CONESUP), UNESCO y el CIESPAL, Quito, 23-24 de julio, 2002.

GONZÁLEZ, A. y SAMPER, Y. Iniciativa municipal para el desarrollo local. Tesis de grado, Facultad de Economía, Universidad de la Habana, Ciudad de la Habana, 2006.

GUCID. Editorial. Boletín del Programa Ramal: Gestión Universitaria del Conocimiento y la Innovación para el Desarrollo. Año I No. 3 noviembre, 2010.

GUCID. Gestión municipal para el desarrollo: estrategias, instrumentos, estilos y métodos. Taller Internacional "Desarrollo Local en Municipios de Ecosistemas frágiles". Santiago de Cuba, 21 al 24 de septiembre, 2004.

GUZÓN, A. "Potencialidades de los municipios cubanos para el desarrollo local". Tesis de Maestría. Edición Ligera. Ciudad Habana, 2003.

NACIONES UNIDAS. Programa Conjunto "Apoyo a las nuevas iniciativas de descentralización y estímulo productivo en Cuba” Ventanilla Temática Desarrollo y Sector Privado. 2009.

NÚÑEZ. J. "La gestión del conocimiento, la Ciencia y la Tecnología en La Nueva Universidad: una aproximación conceptual" con L. F. Montalvo e I. Pérez Ones, en: La Nueva Universidad Cubana y su contribución a la universalización del conocimiento; Hernández, D y Benítez, F (Comp.), Editorial Félix Varela, La Habana, 2006. 